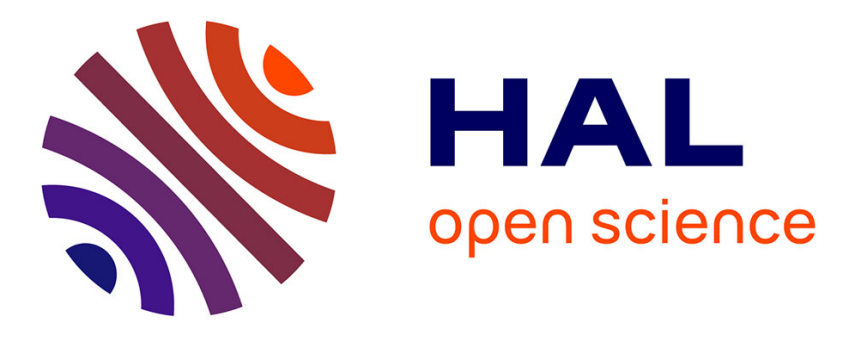

\title{
Delamination and wrinkling of flexible conductive polymer thin films
}

Kaili Xie, Alizée Glasser, Shekhar Shinde, Zaicheng Zhang, Jean-Michel

Rampnoux, Abdelhamid Maali, Eric Cloutet, Georges Hadziioannou, Hamid Kellay

\section{To cite this version:}

Kaili Xie, Alizée Glasser, Shekhar Shinde, Zaicheng Zhang, Jean-Michel Rampnoux, et al.. Delamination and wrinkling of flexible conductive polymer thin films. Advanced Functional Materials, In press, 10.1002/adfm.202009039 . hal-03154867

\section{HAL Id: hal-03154867 \\ https://hal.science/hal-03154867}

Submitted on 1 Mar 2021

HAL is a multi-disciplinary open access archive for the deposit and dissemination of scientific research documents, whether they are published or not. The documents may come from teaching and research institutions in France or abroad, or from public or private research centers.
L'archive ouverte pluridisciplinaire HAL, est destinée au dépôt et à la diffusion de documents scientifiques de niveau recherche, publiés ou non, émanant des établissements d'enseignement et de recherche français ou étrangers, des laboratoires publics ou privés. 


\title{
Delamination and wrinkling of flexible conductive polymer thin
}

\section{2 films}

3 Kaili Xie, Alizée Glasser, Shekhar Shinde, Zaicheng Zhang, Jean-Michel Rampnoux, Abdelhamid Maali, 4 Eric Cloutet, Georges Hadziioannou, Hamid Kellay*

${ }_{5}$ Dr. K. Xie, Dr. A. Glasser, Z. Zhang, Dr. J. M. Rampnoux, Dr. A. Maali, Prof. H. Kellay

- Univ. Bordeaux, CNRS, LOMA UMR 5798, F-33405 Talence, France

, Email Address: hamid.kellay@u-bordeaux.fr

Dr. K. Xie, Dr. A. Glasser, Dr. S. Shinde, Dr. E. Cloutet, Prof. G. Hadziioannou

- Univ. Bordeaux, CNRS, Bordeaux INP, LCPO UMR 5629, F-33615, Pessac, France

Keywords: conductive polymer, flexible thin films, wrinkling instability, delamination and adhesion

\begin{abstract}
Polymer based conductive and transparent thin films are an important class of functional materials at the heart of flexible organic electronic devices. These flexible films are prone to degradation and to mechanical instability leading to the formation of blisters, wrinkles and cracks. This is detrimental to their use especially in the case of multilayer devices. Here, we show that a simple water or solvent drop deposited on such films gives rise to a buckling instability and the formation of several folds due to the tendency of these films to swell in contact with the solvent. A phase diagram of the instability portraying its domain of existence, and thus the means to inhibit it, is proposed. By depositing drops on such films and observing the instability, material parameters such as the elastic modulus of the thin films or their energy of adhesion to the substrate can be estimated reliably. Further, the instability can be harnessed to pattern surfaces at low cost giving rise to percolated and more conductive pathways in the conductive polymer films under scrutiny.
\end{abstract}

\section{Introduction}

Thin films of conductive polymers such as poly(3,4-ethylenedioxythiophene):polyelectrolytes (PEDOT: polyelectrolytes), have received much attention recently due to their flexibility, low sheet resistance, and good optical properties $[1,2]$. They are widely used as electrode layers in electronic devices such as organic light-emitting diodes (OLEDs) [3, 4], organic photovoltaics (OPVs) [5], energy storage cells [6] and hold much promise in the manufacturing of flexible, all organic, devices and sensors. Another advantage of these polymers is that they are water soluble and can be used as water based inks for various deposition and coating purposes. The most widely used polyelectrolyte to stabilize the PEDOT in water is polystyrenesulfonic acid (PSS). An alternative approach [7] replaces PSS with (trifluoromethylsulfonyl)imide (TSFI) side groups attached to a polystyrene backbone (Figure 1a), poly(4-styrene trifuoromethyl (bissulfonylimide)) (PSTFSIK) (see Methods). The presence of hydrogen bonds in the PSTFSI polyanion simplifies the formulation of the conductive inks for various coating and thin film making processes such as doctor blading, inkjet and screen printing [8]. Stacking printed thin films layer by layer 
is a low cost and frequently-used technique to build structured devices with the desired electronic properties and functionalities $[9,10]$. The mechanical stability and adhesion between the adjacent layers is key in the performance and lifetime of such devices [11, 12]. Thin flexible films are, however, prone to a number of instabilities: cracks [13,14], tears [15], or delamination and wrinkling [16, 17]. This is a limiting factor for the performance of devices and a nuisance which needs to be avoided. On other occasions, however, this behavior can be harnessed to achieve additional functionality such as surface patterning at low cost [18-21] or to estimate the properties of materials [15, 17, 22]. Instabilities of thin films can be due to a variety of sources. For example, they may wrinkle or blister in the presence of a mismatch stress because of residual stresses [23], shrinking [24] or swelling as in the case of solvent-responsive polymers $[25-28]$.

Here, we report a spontaneous wrinkling instability of organic flexible conductive thin polymer films initially deposited on rigid substrates. By simply depositing liquid drops, e.g. water, on the films, welldefined microscale wrinkles can be obtained due to swelling. By focusing on the properties of this instability and using a simple model, we rationalize the phase diagram of the instability and explain its main features. Our study offers a simple approach to avoid or harness such instabilities as well as a reliable one for the measurement of material properties, notably, the adhesion energies of thin films on different substrates.

\section{Wrinkling phase diagram}

Thin films of the water based polymer PEDOT:poly(4-styrene trifluoromethyl(bissulfonylimide)) (PEDOT:PSTFSI) [7, 29] (Figure 1a) are deposited onto glass substrates by a Doctor Blade technique [8] (Figure 1b). These films are dried at different temperatures and for different periods of time to evaporate the solvent. The prepared films are then brought naturally to room temperature $\left(21^{\circ} \mathrm{C}\right)$ before additional characterizations are carried out. These films are robust and remain intact when folded (Figure 1b). However, when a solvent drop, e.g. water, is deposited onto the film at room temperature, either the film remains intact or a wrinkling instability occurs. When the wrinkling sets in, random wrinkles are observed in the area close to the center of the drop while finger-like wrinkles are observed near the contact line or front of the drop (Figure 1c and Video S1 in Supplementary Information). These fingerlike wrinkles near the edge of the drop grow preferentially perpendicular to the contact front and their number $N$ depends on drop radius $R_{s}$ (micrographs in Figure 1c). Whether wrinkles arise depends on film preparation and in particular its drying conditions. This drying is used to evaporate the solvent remaining in the films. Figure 1d summarizes the instability phase diagram. Films dried at temperatures below $180^{\circ} \mathrm{C}$ always exhibit wrinkling, even though the drying time is as long as 90 minutes. By contrast, the films remain stable when the drying temperature is higher than $260^{\circ} \mathrm{C}$. In the intermediate 
temperature range, the morphology depends on the drying time: Wrinkling is inhibited for longer drying times for example.

\section{Dynamics of wrinkling}

We now turn to the dynamics of the wrinkling. When a water drop with controlled size is deposited, it spreads with the appearance of spontaneous wrinkling (see Video S1 in Supplementary Information). During the spreading, its radius $R_{s}$ and the number of wrinkles $N$ change with time. Four stages can be defined (Figure 2a). In the early period, both the spreading radius and the number of wrinkles increase with time (denoted as Stage I). After this phase, a stationary regime (Stage II) sets in where the number of wrinkles as well as the radius of the drop remain roughly constant. This stationary state is a consequence of the maximum spreading of the drop. After this stage, the evaporation of the water sets in along with a decrease of the number of wrinkles while their local amplitude increases (Stage III). The final stage associated with the full drying of the drop leads to the formation of permanent folds (Stage IV). During the evaporation phase (Stage III), the structure coarsens: the initial small amplitude wrinkles disappear and larger amplitude ones become predominant [28]. These large ridges or wrinkles end up collapsing to give rise to the folds observed in Stage IV. These observations recall the delamination and self-folding observed in other types of thin films such as PDMS films [28] and hydrogel films [30]. The final thickness (far away from the folds) in the central area, previously covered by the drop, is smaller than the initial film thickness which indicates that the film has been stretched (section S1 in Supplementary Information). This alternatively confirms that the formation of the wrinkles and eventual folds [31] is due to the stretching and increase in area of the initial film under the drop. In what follows we will focus on the stationary regime where both the number of wrinkles and the area of the drop are stationary. Our main concern is to understand what sets the number of wrinkles and what determines whether the instability sets in or not.

In the stationary regime, Figure $2 \mathrm{~b}, 2 \mathrm{c}$ and $2 \mathrm{~d}$ show a simplified schematic as well as the properties of the wrinkling pattern. In experiments, drops with different sizes have been deposited on films of different thicknesses. For each drop, once the stationary state has been reached, we measure the number of wrinkles near the front of the drop (see Figure 1c and section S2 in Supplementary Information). A measurement of the number of wrinkles along the perimeter of the drop gives an estimate of the wavelength of the wrinkling instability. The number of well-defined wrinkles has a linear relationship with the drop radius $R_{s}$ (Figure 2d). This linear relation assures that the wavelength of the instability is invariant as the drop diameter changes. However, as the thickness of the film increases, the slope of the plot of $R_{s}$ versus $N$, increases signalling that the wavelength $\lambda \sim R_{s} / N$, increases. Thus, the wavelength of the wrinkling pattern is independent of the drop size, but it increases with the film thickness. Previous work [32] has 


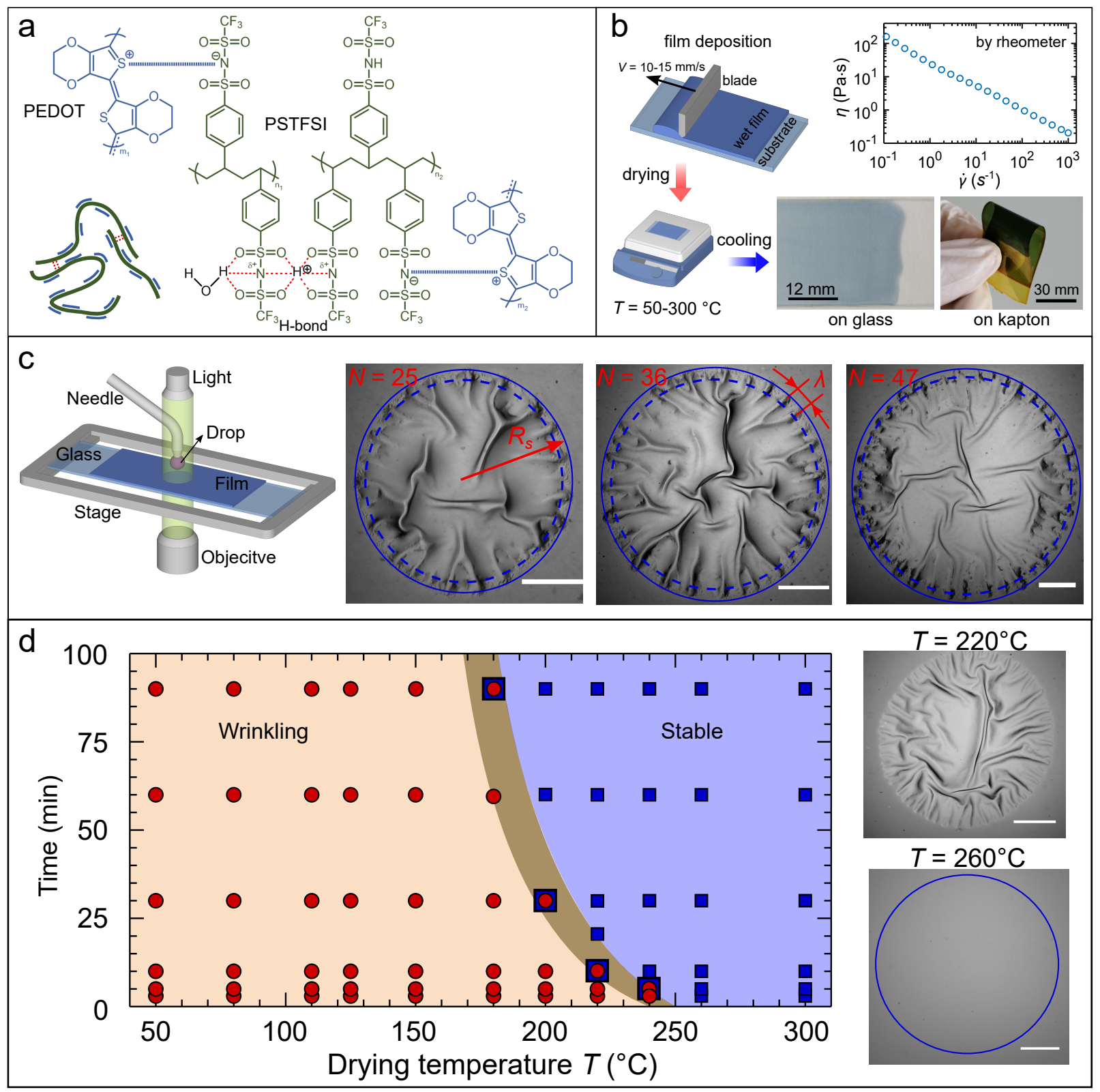

Figure 1: Thin films preparation and wrinkling phase diagram. a, Chemical structure of the conductive polymer PEDOT:PSTFSI. The red dashed lines indicate probable hydrogen bonds. b, Film preparation: doctor-blade coating, drying on the hot plate and cooling to room temperature. Viscosity is measured by a shear rheometer (AR1000, TA Instruments). c, Radial organization of wrinkling. Water drops are deposited via a needle, and an inverted microscope is used to visualize the wrinkling patterns. The images show an increase of the number of wrinkles $N$ counted near the edge of the drop, indicated by the dashed line, with the drop size $R_{s}$. The average spacing between finger-like wrinkles is desig-nated as $\lambda$. The outer full circle indicates the edge of the drop while the dashed circle near the edge indicates the position where the number of wrinkles was counted. Scale bars: $500 \mu \mathrm{m}$. d, Phase diagram of surface stability of films dried at different temperatures and times. The shaded region indicates the transition zone between wrinkle formation and stable films. The presence of superimposed symbols (squares and circles) indicates situations where stable or wrinkled films can be obtained for different films suggesting sensitivity to experimental conditions. Two images are shown in the unstable and stable regions. The full circle in the image for $T=260^{\circ} \mathrm{C}$ for which no wrinkles are observed represents the position of the water drop front in the stationary regime. Scale bars: $500 \mu \mathrm{m}$. 
proposed that wrinkling is in part governed by the bending modulus of the film which increases with its thickness. This gives a qualitative explanation of our observations. In order to explain our observations quantitatively, different considerations enter into play.

a

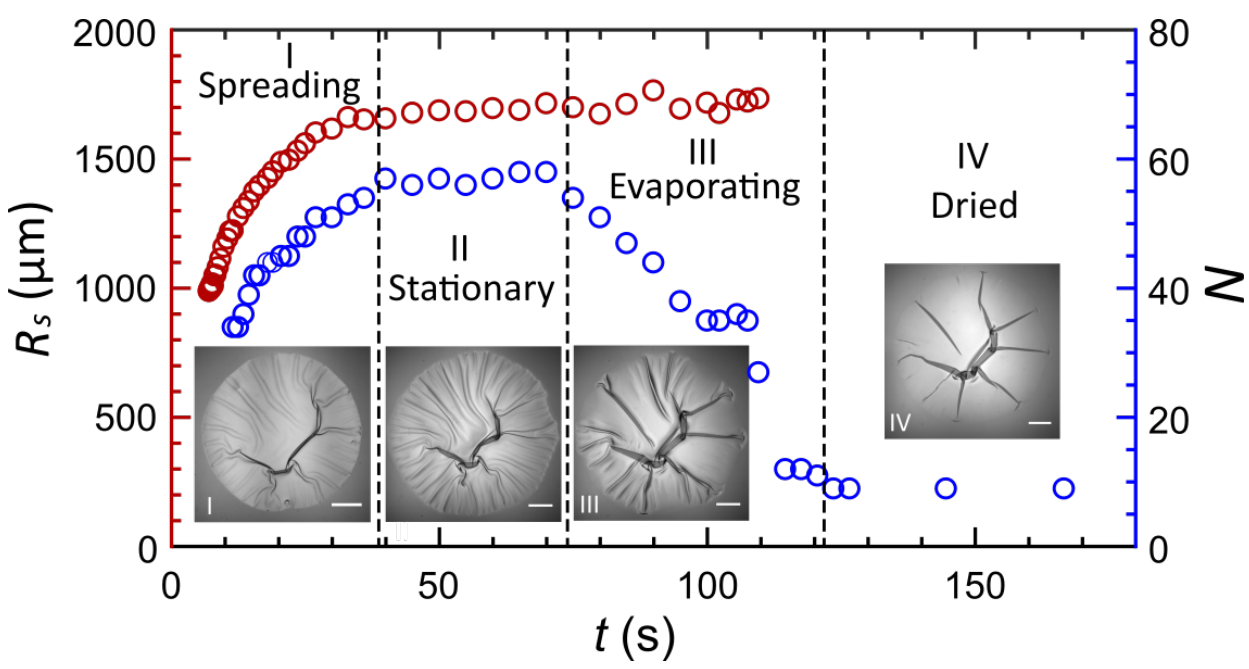

$\mathrm{b}$
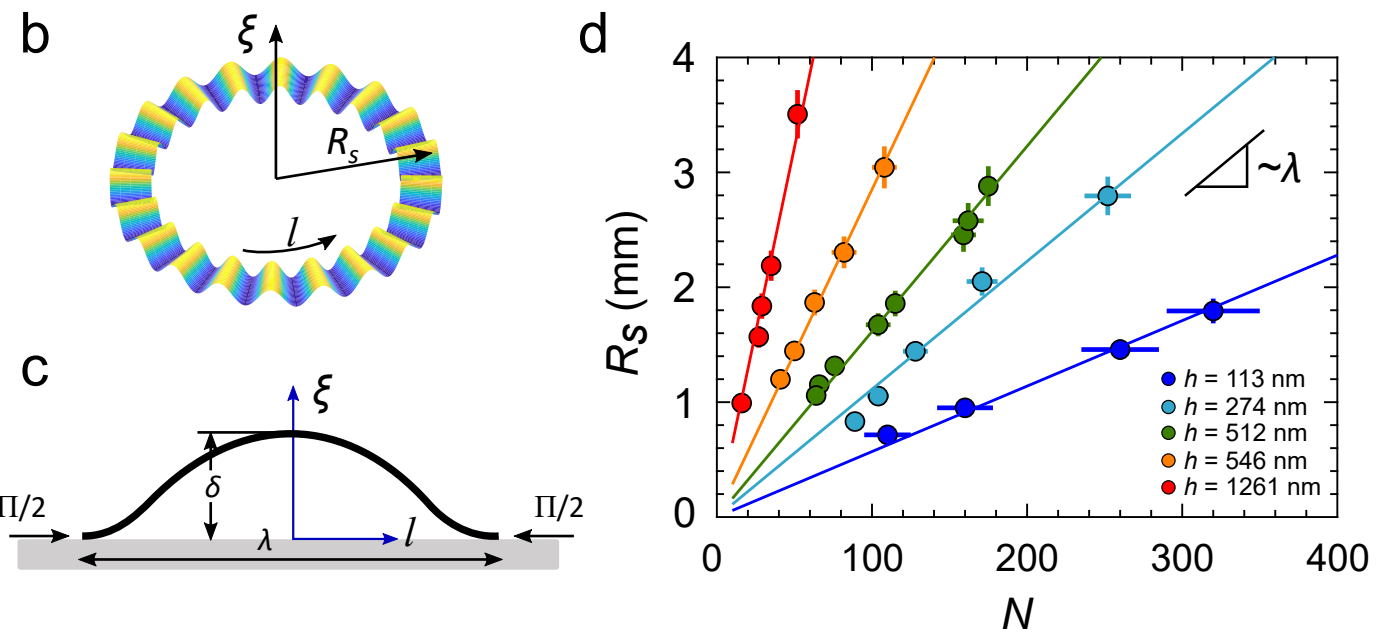

Figure 2: Dynamics and properties of the wrinkling instability. a, Four stages are observed based on the state of the drop, its radius $R_{s}$ and the number of wrinkles $N$ versus time $t$. The number of wrinkles here is counted near the drop front. Insets show images of the wrinkling patterns in the different stages. Scale bar: $500 \mu \mathrm{m}$. b, Schematic of the hypo-thetical three dimensional structure of the well-defined wrinkling pattern near the contact line. c, Hypothetical profile of a single wrinkle with a height $\delta$ and wavelength $\lambda$. The profile along the perimeter $\xi(l)$ is expected to have a cosine shape (Equation S1) under biaxial compression $\Pi$ when the wavelength $\lambda$ is much smaller than the radius $R_{s}$. $\mathbf{d}$, Dependence of the number of wrinkles $N$ versus the radius $R_{s}$ at the front of the drop in the stationary regime. Each data point repre-sents an independent measurement on a drop of radius $R_{s}$. Different drop sizes and film thicknesses are used. Note that since the number of wrinkles measured near the edge of the drop increases linearly with drop radius $R_{s}$, the wavelength of the wrinkling pattern is thus independent of drop size in our observation.

The formation of wrinkles necessarily arises from a competition between the bending of the film, through its bending modulus $B$, and its adhesion to the substrate [17, 33, 34]. The substrate (i.e. glass) is rigid, so for wrinkling to occur the film has to detach from the substrate, a process called delamination. Fur- 
ther, the polymer film used is hygroscopic and swells upon uptake of the solvent [35] as we will see be-low. While we do not fully understand the effect of drying temperature and drying time on film swelling, it has been proposed that high temperatures, apart from removing the residual water, may also affect the conformational state of the PEDOT-polyelectrolyte complex and thus the structure of the film [35-38]. This change may then affect how the film responds to the presence of different liquids such as its own solvent. This swelling increases the area of the film and introduces a mismatch stress between the film under the drop and the rest of the film which remains pinned to the substrate. We hypothesize that this swelling is the driving force for the delamination and the ensuing wrinkling instability. In addition, the drop liquid has to infiltrate beneath the film and wet the substrate and the film, creating two new interfaces, i.e. liquidsubstrate and liquid-film interfaces. The energy cost of this wettability needs to be taken into account [39, 40]. We now propose a simple model taking into account these different contribu-tions to explain the observed wrinkling as well as its properties.

\section{Rationalizing the wrinkling patterns}

To understand the wrinkling and delamination induced by film swelling in our case, we consider a simple scaling approach based on a one-dimensional analysis following previous work on wrinkling [17, 34]. For simplicity, the wrinkling pattern is assumed to be located mainly near the front of the drop and to have a simple periodic functional shape with wavelength $\lambda$ and amplitude $\delta$. The amplitude $\delta$ is assumed to be constant as a function of distance to the center of the drop, at least in the area where we count the wrinkles number. This assumption is difficult to justify a-priori but previous work suggests that this variation is weak [42]. Each wrinkle is also assumed to be under a biaxial compression $\Pi$ (Figure 2c). The film takes up water and swells resulting in a mismatch strain $\varepsilon_{m}$ between the film underneath the drop and the film outside [43] If the mismatch stress is lower than the so called critical stress for de-lamination, the film is expected to remain adhered to the substrate [34]. The energy due to swelling is stored in the plane of the film [25], and once it overcomes the adhesion, delamination takes place releas-ing the stress. The swelling of the film is essential to the development of the instability comes from ad-ditional measurements of the swelling rate of the film (see section S6 in Supplementary Information and Figure 3c). Under the constraint of constant projected surface area, i.e. the area of the drop, the film can only bend out of the plane introducing (for a single wrinkle) a bending energy $U_{b}=B \pi^{4} \delta^{2} / \lambda^{3}$ (see section S3 in Supplementary Information). As mentioned above, the drop liquid fills the delaminated area introducing two additional surface energies i.e. liquid-film $\gamma_{l f}$ and liquid-substrate $\gamma_{l s}$. The net in-terfacial energy change can be written as

$$
U_{i}=\lambda \Delta \gamma,
$$



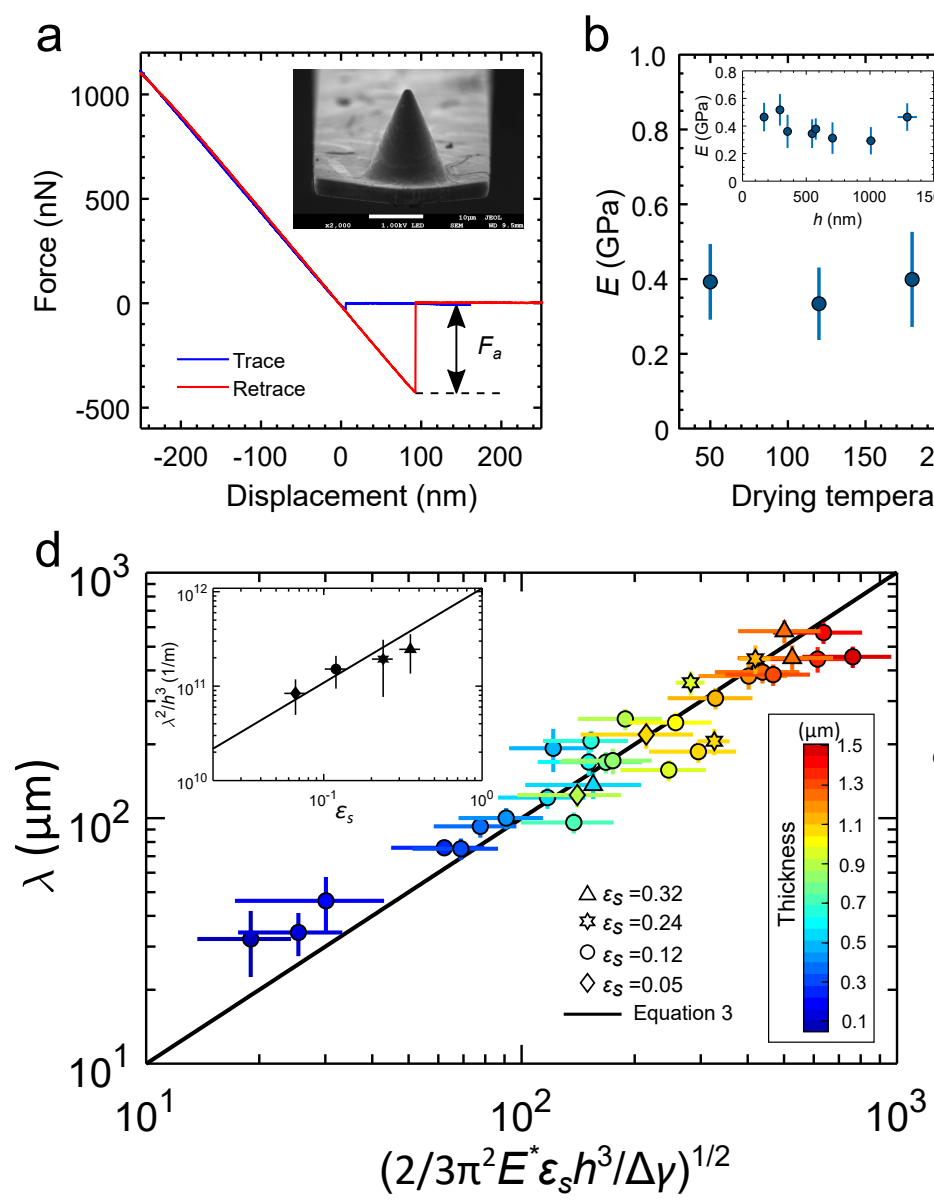

b

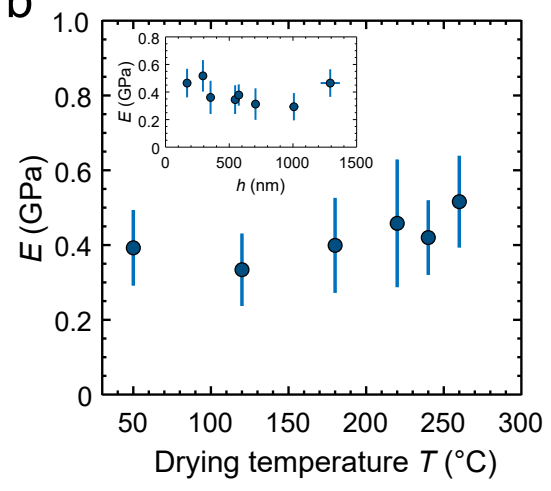

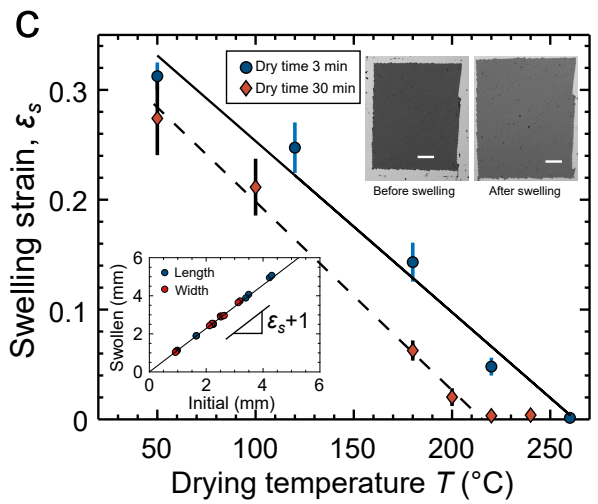

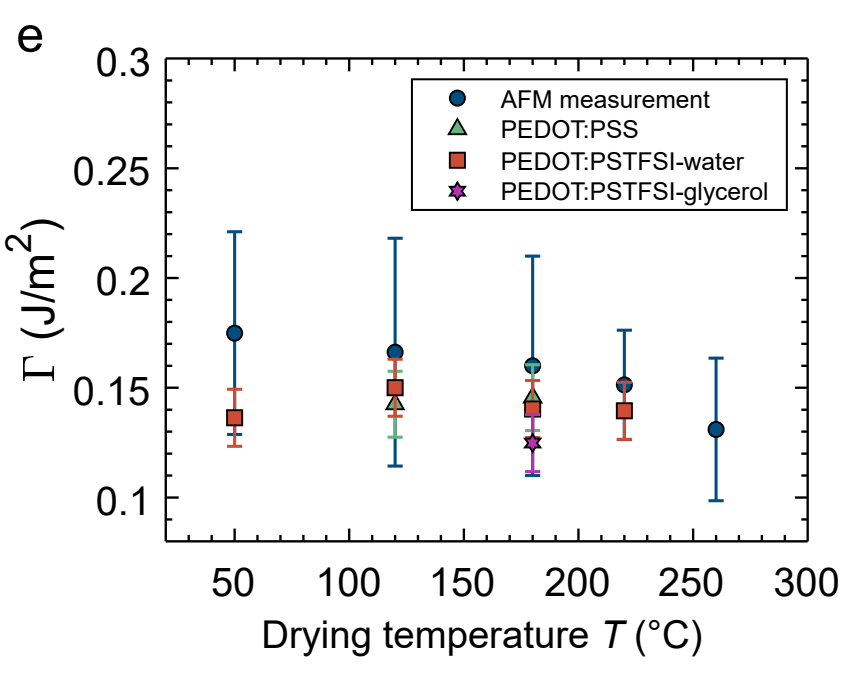

Figure 3: Characterization of the films and wrinkles wavelength. a, An example of a force-displacement curve for trace (approach) and retrace (retraction) from a nano-indentation test by AFM. The Young's modulus can be obtained by fitting the force-indentation curve with the JKR model, and the adhesion force $F_{a}$ can be estimated at the moment of separation between the tip and the film. Inset: SEM image of AFM tip (made of Silicon but covered with an $\mathrm{SiO}_{2}$ layer, $R_{c}=$ $840 \mathrm{~nm})$. b, Young's modulus of the films prepared under different drying temperatures. Inset: Young's modulus of films with different thicknesses. Poisson ratio is assumed as 0.4 here. $\mathbf{c}$, Examples of swelling strain of the films as a function of drying temperature. The films are dried for 3 and 30 minutes for the two cases shown. The strain is obtained by measur-ing the increase in the length of a strip upon water uptake. The strips are rectangular and the inset shows an example of the increase in length and width of these strips (see section S6 in Supplementary Information). Inset images show the film before and after swelling (scale bars are $500 \mu \mathrm{m}$ ). d, Wavelength of wrinkling. Each point here is taken from the slope of $R_{s}$ versus $N$ using 4 to 8 drops as shown in Figure 2d. In total, around 190 drops have been tested. The color code gives the thickness of the films used. Different symbols designate films dried at different temperatures and thus different swelling strains. The inset shows $\lambda^{2} / h^{3}$ versus the strain $\varepsilon_{s}$. The straight line is the prediction of our model using the same param-eters as the main plot. e, Estimates of the adhesion energy. Both water and glycerol drops are used to introduce wrinkling. The data from AFM measurement shown here is for PEDOT:PSTFSI films. The mechanical properties of PEDOT:PSS are taken from Ref. [41].

where $\Delta \gamma=\Gamma-\gamma_{l}\left(\cos \theta_{s}+\cos \theta_{f}\right)$ (see section S3 in Supplementary Information), $\Gamma$ is the adhesion energy, $\gamma_{l}$ is the surface tension of the solvent, and $\theta_{s}$ and $\theta_{f}$ are the contact angles of the solvent on the substrate and the film respectively (see section S8 for measurements of the contact angles). The stiffness 
of the liquid, due to the hydrostatic pressure is neglected as the film is immersed inside the liquid rather than floating [44]. At equilibrium, the swelling energy is expected to be totally released by bending and interfacial energy change, leading to total energy $U_{t}=U_{b}+U_{i}$. By minimizing this energy with respect to the wavelength, $\partial U_{t} / \partial \lambda=0$, we obtain

$$
\lambda^{4}=2 \pi^{4} \frac{B}{\Delta \gamma} \delta^{2},
$$

where the bending modulus of the film $B=E^{*} h^{3} / 12$ with the effective Young's modulus $E^{*}=E /(1-$ $\nu^{2}$ ) using the Young's modulus $E$ and the Poisson ratio $\nu$. The amplitude of wrinkling $\delta$ is too small for measurements using optical methods [17]. On the other hand, the size of the wrinkles is not small enough to use AFM scanning [45]. From the geometrical relationship of the assumed wrinkling profile (Figure 2c), the amplitude $\delta$ can be related to the compressive strain by $\varepsilon_{m}=\Pi / \lambda=\pi^{2} \delta^{2} / 4 \lambda^{2}$, where $\Pi$ is the compression due to film swelling (see section S3 in Supplementary Information). We assume that the mismatch strain is equivalent to the swelling strain of the film, $\varepsilon_{m}=\varepsilon_{s}$ because the swelling energy is totally released. Thus, by replacing the amplitude $\delta$ in Equation 2, we obtain,

$$
\lambda=\left(\frac{2 \pi^{2} E^{*}}{3 \Delta \gamma} \varepsilon_{s} h^{3}\right)^{1 / 2} .
$$

This scaling law is similar to the previously proposed predictions for the wrinkling or buckling wave-length due to a competition between bending and adhesion [17,34]. Our prediction however explicitly takes into account the swelling of the film at the origin of the compression. Note that Equation 3 for the wavelength uses only quantities that can be measured independently and can thus be tested directly. The Young's modulus and, a priori, the energy of adhesion can be obtained by independent measure-ments using AFM nanoindentation [46], as shown in Figure 3a and Figure 3b. The value of Young's mod-ulus $E$ turns out to be near $0.4 \mathrm{GPa}$ with no pronounced dependence on the film preparation temper-ature nor its thickness. The measurements of the adhesion energy on the other hand show much scat-ter from measurement to measurement and from different thicknesses. On average, this adhesion energy hovers around $0.16 \mathrm{~J} / \mathrm{m}^{2}$ with no systematic dependence on the film thickness or drying temperature (see section S7 in Supplementary Information).

The swelling strain can be estimated by independent measurements (see section S6 in Supplementary Information). Figure 3c illustrates that the swelling strain decreases linearly with the drying temperature for a given drying time, and almost zero swelling is detected when the temperature is high. This observation coupled to the prediction of our model explains the fact summarized in the phase diagram (Figure 1d) that the films under high drying temperatures show no buckling: The driving force due to the swelling strain is negligible for these films. Further, the phase diagram indicates that the longer the drying time, the smaller is the temperature for which stable films are obtained. This is in line with our measurements of the swelling strain which decreases as the drying time increases making for more sta- 
ble films at even lower drying temperatures (Figure 3c). In fact, the phase diagram may be related to the existence of a critical strain for instability (see Figure S8) as predicted recently, with the stable film region having strains below a threshold value [34].

In order to quantitatively compare our experimental results for the wavelength from different deposited drops and different film thicknesses with the theoretical prediction, we plot the wavelength $\lambda$ versus the quantity $\left(2 \pi^{2} E^{*} \varepsilon_{s} h^{3} / 3 \Delta \gamma\right)^{1 / 2}$ (Figure $3 \mathrm{~d}$ ). Here, we use the measured values of the strain and the Young's modulus, however, adhesion $\Delta \gamma$ or $\Gamma$, which is difficult to measure with good enough precision, is left as an adjustable parameter. In Figure 3d, the wavelength follows the predicted trend and the best fit cor-responds to a value of $\Gamma=0.138 \mathrm{~J} / \mathrm{m}^{2}$. This is in good agreement with the one estimated using AFM measurements; the difference is roughly 14 percent. A summary of the adhesion energies obtained for different drying temperatures is shown in Figure 3e. The energy of adhesion obtained from the analysis of wrinkling is roughly independent of the drying temperature and is consistent with the values es-timated by AFM nanoindentation. To further test the model, we also plot in the inset to Figure $3 \mathrm{~d}$ the dependence of the wavelength normalized by the thickness of the film, $\lambda^{2} / h^{3}$, on the strain $\varepsilon_{s}$. The agree-ment with the expected result as illustrated by the straight line, using the same parameters as the main figure (Figure 3d), is good indicating that our model captures the essential features of our measurements namely the dependence on the thickness of the film and the dependence on the swelling strain. The pre-diction of the wavelength is thus quantitative and accounts for all the features observed: no dependence of the wavelength on drop radius, sensitivity to film swelling, and good agreement with material parame-ters notably the Young's modulus and the adhesion energy. As mentioned above, our scaling is based on the work of Reference [17, 34], but other models have been proposed to explain the wrinkling or buckling instability of thin films. A comparison of our experimental data to other scaling laws using other models is shown in section S4 in Supplementary Information [47-50]. Because of different geometries and differ-ent driving forces for wrinkling or buckling, these models do not fully explain our experimental findings.

\section{Discussion}

This wrinkling instability using water drops can also be observed for other types of films and using other liquids. For example, the film wrinkling is also observed when glycerol drops are deposited on the film (Figure 4a). Compared with the water drop, the wrinkles are more ordered: straight and point outwards at the front of the drop. However, the emergence of this well ordered wrinkling pattern is much slower than that for water drops due to the difference in viscosity of the two liquids. In another example, we used the commercially available conductive polymer PEDOT:PSS, widely used in many applications $[6$, 

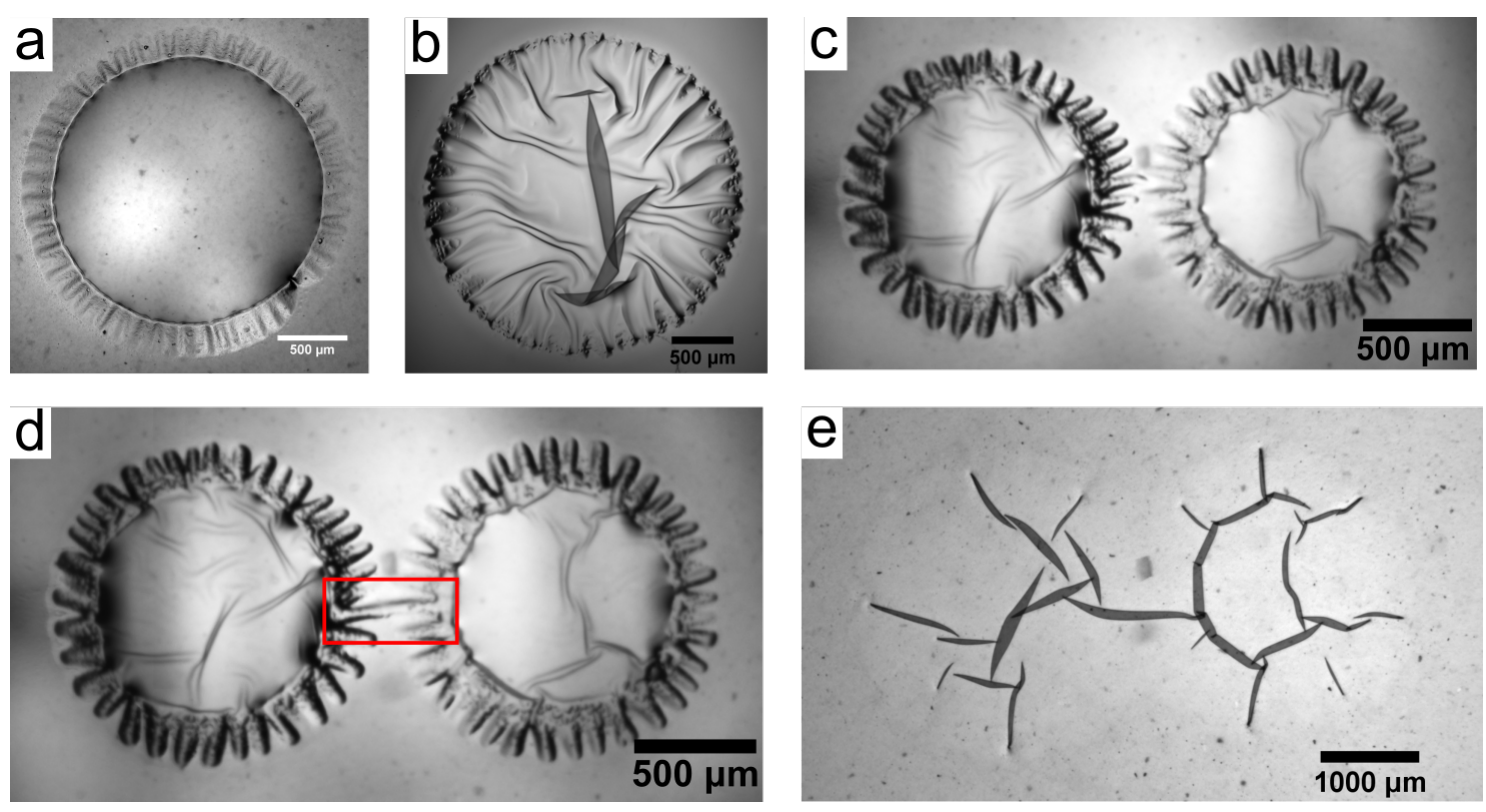

Figure 4: Polymer film surface patterning. a, Periodic wrinkling induced by a glycerol drop on a PEDOT:PSTFSI film. b, Wrinkling pattern on a PEDOT:PSS film using a water drop. c-e, Interaction of wrinkling patterns induced by two water drops. A connection takes place when the wrinkles are close enough (the red rectangle) and it remains after drying.

51]. A closely similar pattern as that obtained with PEDOT:PSTFSI is observed (Figure 4b). Adhesion energies for these two experiments, obtained using the wrinkling pattern, remain in the range of values measured for PEDOT:PSTFSI films (Figure 3e). We are not aware of other measurements of the adhesion energy of PEDOT:polyelectrolytes polymer inks on glass. Nevertheless, the estimated value of $(\Gamma \approx$ $0.14 \mathrm{~J} / \mathrm{m}^{2}$ ) compares favourably at least in order of magnitude with previous measurements on closely related systems [22].

Besides providing a means to measure material properties and surface adhesion in particular, our findings also offer a simple way to pattern thin film surfaces. Figure 4c, 4d and 4e show two nearby drops as well as their respective engendered patterns. The proximity of the two drops leads to a connection between their respective wrinkles: Two neighbouring wrinkles merge with each other forming a connection (Figure 4d and Video S2 in Supplementary Information). As the drops dry, the connection persists making for a connected network of folds (Figure 4e). These folds consist of a few layers of material (section S1 in Supplementary Information). If these layers remain connected, which is the case in this example, a percolated network can then be obtained on the surface of the film if multiple drops are deposited. We speculate that this network will lead to a better conductivity of the film since sheet resistance in the folded area is lower than in the film. For example, four-layer folds can lower the sheet resistance a factor of 6.8 for undoped films whereas the thickness only increases a factor of 4 (see section S5 in Supplementary Information). More electron pathways are thus created by folding. In the meantime, the film maintains its transparency because of the network remaining sparse. 


\section{Conclusion}

In summary, we have described a wrinkling instability of thin polymer films. This instability is engendered by simple contact with a liquid (water) and swells the film giving rise to delamination. The observed wrinkling instability can be completely inhibited by using appropriate preparation: High enough drying temperatures or long drying times prevent the swelling of the film and thus the emergence of such buckling. The well-defined wrinkling patterns near the front of the drop, are rationalized using a simple model. The wavelength of the patterns is related to the film swelling, the mechanical properties of the film and the adhesion energy between the film and substrate. By coupling the model to the observations, the adhesion energy of the film-substrate system, a difficult quantity to measure with good precision but essential in the design of electronic devices, can be estimated reliably. The method is applicable to other types of polymer-substrate systems. Further, we show through an example that this wrinkling can be tamed to produce patterned surfaces with improved conductivity.

\section{Experimental Section}

Materials: Sodium 4-vinylbenzenesulfonate, 2,2'-azobis(2-methylpropionitrile)(AIBN), hydrochloric acid, dichloromethane, potassium carbonate and iron III chloride were purchased from Sigma-Aldrich. Acetone, ethanol, isopropanol, methanol, diethyl ether, dimethyl sulfoxide (DMSO), tetrahydrofuran (THF) and oxalyl chloride were purchased from Sigma-Aldrich as received. N,N-dimethylformamide (DMF) and dry-acetonitrile were purchased from Acros Organics. Sodium bicarbonate, triethylamine, ammonium persulfate and 3,4-Ethylenedioxythiophene (EDOT) were purchased from Fisher Scientific. Trifluoromethanesulfonamide and S-dodecyl-S'-( $\alpha, \alpha^{\prime}$-dimethyl- $\alpha^{\prime \prime}$-acetic acid) trithiocarbonate (CTA) are from ABCR GmbH. Lewatit S100 KR/H and MP 62 WS were purchased from Lanxess. Commercial PEDOT:PSS (Clevios PH500) solution was purchased from Heraeus. Glycerol was purchased from VWR. All products were used without further purification. Water was used from a millipore water system (PureLab Flex).

Polymer synthesis: EDOT was stabilized with potassium salt of polyanion poly(4-styrene trifluoromethyl (bissulfonylimide)) (PSTFSIK) by ionic interaction during the oxidative dispersion polymerization of EDOT in water. The synthesis of PSTFSK followed previous protocols $[7,8,52]$. The yields of monomer STFSIK and polyanion PSTFSK were around $40 \%$ and $50 \%$. Molecular weight of polyanion PSTFSIK used in our study was 100kDa. The complexation between EDOT and PSTFSIK was done according to Ref. 8, 29. For further processing, the ink solution was concentrated with a final concentration of PEDOT around $1.2 \%$ which is very viscous at room temperature.

Substrate preparation: Prior to film deposition, microscope slides (RS France, $26 \mathrm{~mm} \times 76 \mathrm{~mm}$ ) were 
first cleaned with acetone, ethanol and isopropanol in an ultrasonic bath for 10 minutes each and then dried with clean nitrogen. To enhance the wettability, the slides were subsequently treated by UV/ozone for 15 minutes. After cleaning, we used the substrates immediately. The contact angle of liquid on the substrate was measured by static sessile drop method (DSA100, Krüss). For flexible substrates, kapton was cleaned with ethanol and isopropanol, and dried with nitrogen gas before depositing onto a glass substrate. The conductive polymer ink was then coated on the kapton surface.

Film deposition and processing: A rigid doctor blade (Erichsen) was used to deposit the film onto the pre-cleaned substrates (glass or glass covered by kapton). The thickness of the film can be tuned by adjusting the gap between the substrate and the blade and coating speed. Since the PEDOT:PSTFSI solution is shear-thinning (inset in Figure 1b, measured by a rheometer (TA Instruments)), we carefully chose the proper gap and speed. After deposition, the film was dried on a hot plate in ambient atmosphere under different temperatures and for different times in a clean room, followed by cooling to room temperature. The film thickness was then measured by a profilometer (Bruker Dektak) and interferometry (Horiba JY UVISEL) (section S1 in Supplementary Information). The contact angle of the liquid on the film was measured by a static sessile drop method (section S8 in Supplementary Information).

Four-point probe measurement: The sheet resistance of the film was measured by a four-point probe device (Lucas 302). A computer controlled Keithley was used to apply the current (10-100 $\mu \mathrm{A})$ through the two outer probes while the voltage was measured by the two inner probes. The measurement accuracy is around $1 \%$. Several different positions were measured for each sample.

Young's modulus, adhesion and swelling properties measurement: The Young's modulus of the film was estimated by performing nanoindentation measurements using AFM (D3100, Bruker). A silicon cantilever (LRCH 225 C3.0-R, Nanotec GmbH) with a radius of curvature of $840 \mathrm{~nm}$ and spring constant of $4.8 \mathrm{~N} / \mathrm{m}$ was used. Several positions were tested for each sample. Two different ramp sizes, $500 \mathrm{~nm}$ and $1000 \mathrm{~nm}$, were used for each force curve measurement. To fit the force-indentation trace curve, Johnson-Kendall-Robert (JKR) model [53] was considered. In our experiments, all the measurements were conducted at the relative humidity of $45-60 \% \mathrm{RH}$ and room temperature of $21-22{ }^{\circ} \mathrm{C}$.

To estimate the compressive strain due to swelling, we carefully cut the film into rectangular pieces with different sizes (at least 5 different pieces), and immersed the samples into the solvent bath. Once the swelling reaches equilibrium (a few seconds), images were captured by a camera (C11440, Hamamatsu) equipped on a microscope (AX10, Zeiss). The lengths of four sides before and after swelling were measured by ImageJ. The swelling strain is thus calculated as $\varepsilon_{s}=\left(L_{s}-L_{0}\right) / L_{0}$, where $L_{0}$ and $L_{s}$ are the unswollen and swollen lengths, respectively. More details are given in section S6 in Supplementary Information.

Drop deposition: To deposit water drop with controllable size on the film, a needle (320 $\mu \mathrm{m}$ of diameter) 
was connected to a precise syringe pump (Pump 11 Elite, Harvard Apparatus). Once the drop was generated, The needle was lowered slowly to approach the film surface. When the drop touched the surface, the needle was quickly removed out of the observation window, to avoid its influence on visualization (see the videos in Supplementary Information). For glycerol drops, since its viscosity is large, a larger needle size (820 $\mu \mathrm{m}$ in diameter) was used. All images were directly visualized with an inverted microscope (AX10, Zeiss) equipped with a high resolution camera (C11440, Hamamatsu). Image processing was done by a home-made Matlab program.

\section{Supporting Information}

Supporting Information is available from the Wiley Online Library or from the corresponding author.

\section{Acknowledgements}

The authors gratefully acknowledge funding support from LabEx AMADEUS ANR-10-LABEX-0042AMADEUS, the program of Excellence Initiative IdEx ANR-10-IDEX-003-02, ANR MULTISPOT ANR17-CE09-0015. K.Xie thanks S. Khiev for SEM measurement, D. P. Mantione for fruitful discussion, and M. Bousquet for the help of polymer synthesis. The authors also thank the machine shop at LOMA for help with experiment setup. H. Kellay acknowledges support from Institut Universitaire de France.

\section{References}

[1] J.-S. Yeo, J.-M. Yun, D.-Y. Kim, S. Park, S.-S. Kim, M.-H. Yoon, T.-W. Kim, S.-I. Na, ACS Applied Materials and Interfaces 2012, 4, 52551.

[2] S. De, P. E. Lyons, S. Sorel, E. M. Doherty, P. J. King, W. J. Blau, P. N. Nirmalraj, J. J. Boland, V. Scardaci, J. Joimel, et al., ACS Nano 2009, 3, 3714.

[3] A. I. Hofmann, E. Cloutet, G. Hadziioannou, Advanced Electronic Materials 2018, 4, 101700412.

[4] A. P. Kulkarni, C. J. Tonzola, A. Babel, S. A. Jenekhe, Chemistry of Materials 2004, 16, 234556.

[5] H. Youn, H. J. Park, L. J. Guo, Small 2015, 11, 192228.

[6] S.-I. Na, S.-S. Kim, J. Jo, D.-Y. Kim, Advanced Materials 2008, 20, 214061.

[7] A. I. Hofmann, W. T. Smaal, M. Mumtaz, D. Katsigiannopoulos, C. Brochon, F. Schütze, O. R. Hild, E. Cloutet, G. Hadziioannou, Angewandte Chemie 2015, 127, 298626.

[8] A. Glasser, E. Cloutet, G. Hadziioannou, H. Kellay, Chemistry of Materials 2019, 31, 176936.

[9] T. Sharma, S.-S. Je, B. Gill, J. X. Zhang, Sensors and Actuators A: Physical 2012, 17787.

[10] C. Wu, F. Li, T. Guo, T. W. Kim, Organic Electronics 2012, 13, 1178. 
[11] B. Wei, J. Liu, L. Ouyang, C. C. Kuo, D. C. Martin, ACS Applied Materials and Interfaces 2015, 7, 2815388.

[12] L. Ouyang, B. Wei, C.-c. Kuo, S. Pathak, B. Farrell, D. C. Martin, Science Advances 2017, 3, 3 e1600448.

[13] Y.-Y. Lee, J.-H. Lee, J.-Y. Cho, N.-R. Kim, D.-H. Nam, I.-S. Choi, K. T. Nam, Y.-C. Joo, Advanced Functional Materials 2013, 23, 324020.

[14] D. J. Lipomi, J. A. Lee, M. Vosgueritchian, B. C. K. Tee, J. A. Bolander, Z. Bao, Chemistry of Materials 2012, 24, 2373.

[15] E. Hamm, P. Reis, M. LeBlanc, B. Roman, E. Cerda, Nature Materials 2008, 7, 5386.

[16] X. Yang, Y. Zhao, J. Xie, X. Han, J. Wang, C. Zong, H. Ji, J. Zhao, S. Jiang, Y. Cao, et al., ACS Nano 2016, 10, 33801.

[17] D. Vella, J. Bico, A. Boudaoud, B. Roman, P. M. Reis, Proceedings of the National Academy of Sciences 2009, 106, 2710901.

[18] Y. Sun, W. M. Choi, H. Jiang, Y. Y. Huang, J. A. Rogers, Nature Nanotechnology 2006, 1, 3201.

[19] F. Tardani, W. Neri, C. Zakri, H. Kellay, A. Colin, P. Poulin, Langmuir 2018, $34,92996$.

[20] A. Deblais, R. Harich, D. Bonn, A. Colin, H. Kellay, Langmuir 2015, 31, 225971.

[21] A. Deblais, R. Harich, A. Colin, H. Kellay, Nature Communications 2016, 7, 11.

[22] J. Ma, D. G. Cahill, N. Miljkovic, Nano Letters 2020, 20, 53918.

[23] A. Pundt, E. Nikitin, P. Pekarski, R. Kirchheim, Acta Materialia 2004, 52, 61579.

[24] J. Kim, S.-J. Park, T. Nguyen, M. Chu, J. D. Pegan, M. Khine, Applied Physics Letters 2016, 108, 6061901.

[25] A. Pandey, D. P. Holmes, Soft Matter 2013, 9, 235524.

[26] H. S. Kim, A. J. Crosby, Advanced Materials 2011, 23, 364188.

[27] H. Vandeparre, P. Damman, Physical Review Letters 2008, 101, 12124301.

[28] S. S. Velankar, V. Lai, R. A. Vaia, ACS Applied Materials and Interfaces 2012, 4, 124.

[29] A. I. Hofmann, D. Katsigiannopoulos, M. Mumtaz, I. Petsagkourakis, G. Pecastaings, G. Fleury, C. Schatz, E. Pavlopoulou, C. Brochon, G. Hadziioannou, E. Cloutet, Macromolecules 2017, 50, 51959. 
[30] B. B. Xu, Q. Liu, Z. Suo, R. C. Hayward, Advanced Functional Materials 2016, 26, 193218.

[31] K. Lee, S. Lee, D.-Y. Khang, T. Lee, Soft Matter 2010, 6, 14.

[32] E. Cerda, L. Mahadevan, Physical Review Letters 2003, 90, 7074302.

[33] S. Edmondson, K. Frieda, J. E. Comrie, P. R. Onck, W. T. Huck, Advanced Materials 2006, 18, 6 724.

[34] B. Davidovitch, V. Démery, arXiv preprint arXiv:2007.04748 2020.

[35] J. Zhou, D. H. Anjum, L. Chen, X. Xu, I. A. Ventura, L. Jiang, G. Lubineau, Journal of Materials Chemistry C 2014, 2, 469903.

[36] X. Fan, W. Nie, H. Tsai, N. Wang, H. Huang, Y. Cheng, R. Wen, L. Ma, F. Yan, Y. Xia, Advanced Science 2019, 6, 191900813.

[37] U. Lang, E. Müller, N. Naujoks, J. Dual, Advanced Functional Materials 2009, 19, 81215.

[38] E. Vitoratos, S. Sakkopoulos, E. Dalas, N. Paliatsas, D. Karageorgopoulos, F. Petraki, S. Kennou, S. A. Choulis, Organic Electronics 2009, 10, 161.

[39] A. Gent, J. Schultz, The Journal of Adhesion 1972, 3, 4281.

[40] D. A. Sanchez, Z. Dai, P. Wang, A. Cantu-Chavez, C. J. Brennan, R. Huang, N. Lu, Proceedings of the National Academy of Sciences 2018, 115, 317884.

[41] U. Lang, N. Naujoks, J. Dual, Synthetic Metals 2009, 159, 5-6 473.

[42] Z. Dai, D. A. Sanchez, C. J. Brennan, N. Lu, Journal of the Mechanics and Physics of Solids 2020, $13 \%$

[43] L. B. Freund, S. Suresh, Thin film materials: stress, defect formation and surface evolution, Cambridge University Press, 2004.

[44] D. Vella, B. Davidovitch, Physical Review E 2018, 98, 1013003.

[45] S. Deng, E. Gao, Z. Xu, V. Berry, ACS Applied Materials and Interfaces 2017, 9, 87812.

[46] M. E. Dokukin, I. Sokolov, Langmuir 2012, 28, 4616060.

[47] B. Davidovitch, R. D. Schroll, D. Vella, M. Adda-Bedia, E. A. Cerda, Proceedings of the National Academy of Sciences 2011, 108, 4518227.

[48] H. King, R. D. Schroll, B. Davidovitch, N. Menon, Proceedings of the National Academy of Sciences 2012, 109, 259716. 
[49] L. Pocivavsek, R. Dellsy, A. Kern, S. Johnson, B. Lin, K. Y. C. Lee, E. Cerda, Science 2008, 320, 5878912.

[50] J. Y. Chung, A. J. Nolte, C. M. Stafford, Advanced Materials 2011, 23, 3349.

[51] S. Ouyang, Y. Xie, D. Wang, D. Zhu, X. Xu, T. Tan, J. DeFranco, H. H. Fong, Journal of Polymer Science Part B: Polymer Physics 2014, 52, 181221.

[52] R. Meziane, J.-P. Bonnet, M. Courty, K. Djellab, M. Armand, Electrochimica Acta 2011, 5714.

[53] K. L. Johnson, K. Kendall, a. Roberts, Proceedings of the Royal Society of London. A. Mathematical and Physical Sciences 1971, 324, 1558301.

Table of Contents

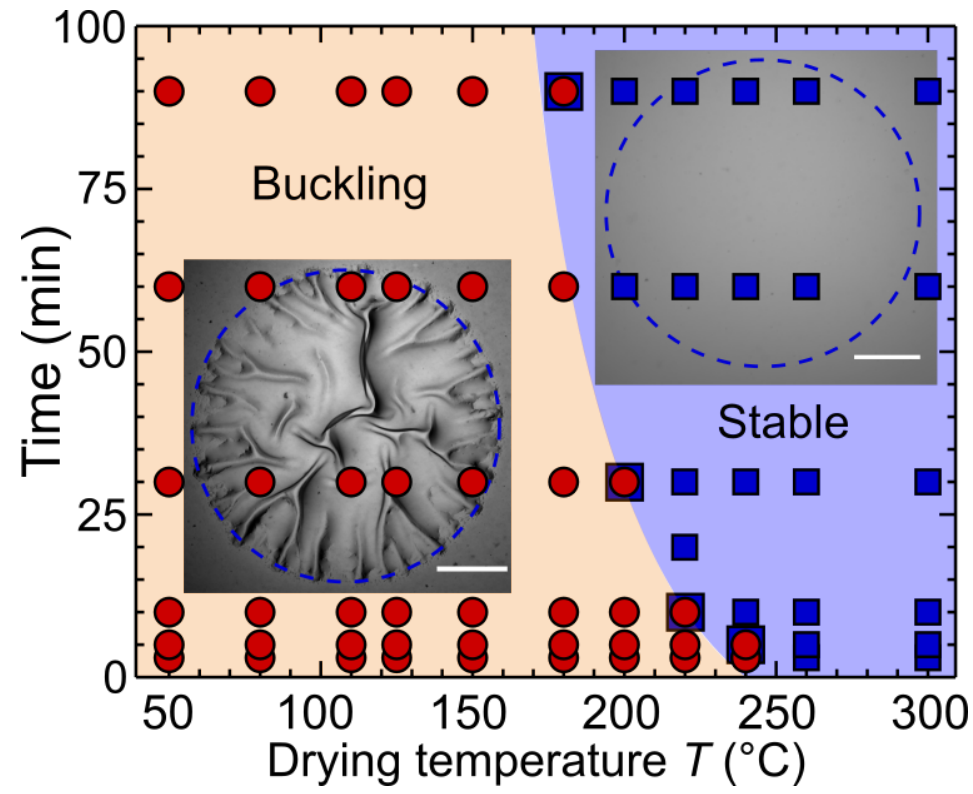

Mechanical stability is essential in thin polymer functional films. A simple water drop deposited on the thin film gives rise to a wrinkling instability. Swelling of the film significantly governs this instability. The phase diagram of the instability allows to control the quality of the films. 